\title{
A Successful Case of Transcatheter Arterial Embolization with N-butyl-2-cyanoacrylate for Pancreatic Arteriovenous Malformation
}

\author{
Tetsuya Tatsuta ${ }^{1}$, Tetsu Endo ${ }^{1}$, Kiyotaka Watanabe ${ }^{1}$, Keisuke Hasui ${ }^{1}$, Naoya Sawada ${ }^{1}$, \\ Go Igarashi ${ }^{1}$, Kenichiro Mikami ${ }^{1}$, Koichi Shibutani ${ }^{2}$, Fumiyasu Tsushima ${ }^{2}$, \\ Yoshihiro Takai ${ }^{2}$ and Shinsaku Fukuda ${ }^{1}$
}

\begin{abstract}
A 57-year-old man was referred and admitted to our hospital for treatment of a symptomatic pancreatic mass. Pancreatic arteriovenous malformation (AVM) was diagnosed based on the findings of contrastenhanced computed tomography (CT) and angiography, and transcatheter arterial embolization (TAE) with $\mathrm{N}$ butyl-2-cyanoacrylate (NBCA) was performed without complications. The patient's symptoms subsequently improved after TAE, and resolution of the pancreatic AVM was detected on contrast-enhanced CT performed six months after the embolization procedure. This case indicates that TAE with NBCA is a safe and effective treatment for pancreatic AVM.
\end{abstract}

Key words: pancreatic arteriovenous malformation, transcatheter arterial embolization, N-butyl-2-

cyanoacrylate

(Intern Med 53: 2683-2687, 2014)

(DOI: 10.2169/internalmedicine.53.3327)

\section{Introduction}

Arteriovenous malformation (AVM) of the pancreas is an extremely rare condition. Most patients present with complications such as abdominal pain, signs of acute pancreatitis, gastrointestinal bleeding and portal hypertension $(1,2)$. Surgical resection is considered to be a radical treatment for pancreatic AVM; however, transcatheter arterial embolization (TAE) has been performed as an alternative approach in recent years (3-5). We herein report the case of a patient with pancreatic AVM who was successfully treated with TAE using means of N-butyl-2-cyanoacrylate (NBCA).

\section{Case Report}

A 57-year-old man was referred and admitted to our hospital for a further evaluation of a symptomatic pancreatic mass suggestive of AVM. Three months before the referral, he had experienced severe upper abdominal pain and a backache. Computed tomography (CT) revealed the conglomeration of strong nodular staining in the pancreatic body and tail in the arterial phase (Fig. 1). Therefore, the patient was referred to our hospital for further investigation and management. He had been diagnosed one year previously with ischemic heart disease, for which he was currently under treatment. However, he had no relevant past history, such as abdominal trauma, pancreatitis or other congenital abnormalities. On a physical examination, no particular findings were noted, except for upper abdominal tenderness, and the laboratory data showed a slight increase in the HbAlc level (6.7\%; reference range: 4.7-6.2\%). Other parameters, including the levels of pancreatic enzymes and tumor markers, were unremarkable, and imaging tests showed no lesions suggestive of AVMs in any organs except for the pancreas.

${ }^{1}$ Department of Gastroenterology and Hematology, Hirosaki University Graduate School of Medicine, Japan and ${ }^{2}$ Department of Radiology, Hirosaki University Graduate School of Medicine, Japan

Received for publication May 20, 2014; Accepted for publication July 21, 2014

Correspondence to Dr. Kenichiro Mikami, kmikami@cc.hirosaki-u.ac.jp 


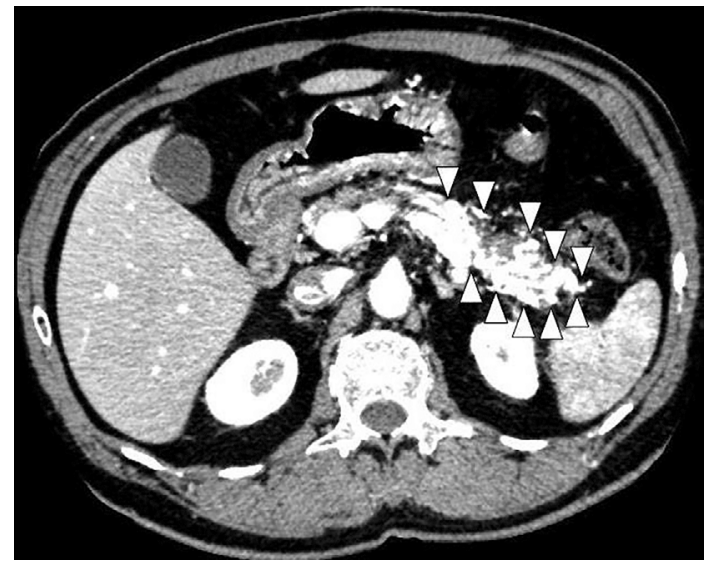

Figure 1. Arterial phase contrast-enhanced CT showed the conglomeration of strong nodular stains in the arterial phase in the pancreatic body and tail (arrowheads).

Esophagogastroduodenoscopy and a barium enema were negative for varices, mucosal lesions and tumors. In order to exclude the possibility of referred pain from ischemic heart disease, coronary angiography was performed, which showed $90 \%$ occlusion of the left anterior descending coronary artery. Therefore, percutaneous coronary intervention (PCI) was subsequently conducted. However, following revascularization with PCI, the patient's abdominal pain persisted, being relieved only slightly with opioids. Accordingly, we considered the abdominal pain to be caused by a pancreatic mass suggestive of AVM, which required angiography for confirmation.

The gastroenterologist, surgeons and interventional radiologists discussed the various therapeutic approaches. In general, surgical resection is the definitive treatment for pancreatic AVM. However, we determined that surgery would not be suitable for the patient, as he had just undergone treatment with PCI for ischemic heart disease. Therefore, TAE was proposed and accepted by the patient as an alternative to surgical intervention.

A diagnosis of pancreatic AVM was made on angiography, and TAE was subsequently performed. Angiography of the celiac artery showed racemose vascular networks in the body and tail of the pancreas, with an early venous return to the portal vein (Fig. 2A). Via superselective microcatheterization, the branches of the dorsal and transverse pancreatic arteries were embolized with the injection of 0.2 and 0.4 $\mathrm{mL}$ of $25 \%$ NBCA, respectively. Although angiography performed after embolization showed a residual pancreatic AVM, no early venous return to the portal vein was detected (Fig. 2B). Three days after the TAE procedure, a repeat CT scan confirmed the presence of embolic material within the pancreatic AVM. Thereafter, the patient had an uneventful clinical course following embolization, and his abdominal pain ceased completely without treatment with opioids. He was subsequently discharged 20 days after the procedure and currently remains asymptomatic with no residual AVMs in the pancreas on CT scans during six months of follow-up
(Fig. 3).

\section{Discussion}

Pancreatic AVM was first reported by Halpern et al. in 1968 and is defined as a tumorous formation or vascular anomaly that shunts the blood flow between the arterial and venous systems in the pancreas (6). An increasing number of cases of pancreatic AVM have been diagnosed due to improvements in imaging modalities. Nevertheless, pancreatic AVM is still considerably rare worldwide, with fewer than 100 cases $(1,7-10)$ reported prior to 2014. The origin of pancreatic AVM may be congenital, as a remnant of the fetal vascular system, or acquired, as a consequence of inflammation, neoplasms or trauma. Ninety percent of pancreatic AVM cases are congenital, with approximately $10-30 \%$ of cases being associated with Osler-Weber-Rendu (OWR) disease, an autosomal dominant syndrome characterized by the presence of mucocutaneous or visceral telangiectasia and AVMs in multiple organs (11). In the present case, the pancreatic AVM was thought to be congenital due to the absence of acquired factors, such as pancreatitis, neoplasms or trauma, as well as a family history and symptoms of OWR disease. Most cases of pancreatic AVMs are symptomatic, and common symptoms include abdominal pain and upper gastrointestinal bleeding, which may arise from esophageal or gastric varices due to portal hypertension (1). The origin of abdominal pain is unknown, although it may be caused by ischemia, the so-called steal syndrome, with shunting of the blood flow into the AVM from the mesenteric circulation (12).

The diagnosis is usually confirmed on imaging studies, such as CT, magnetic resonance imaging (MRI), Doppler ultrasonography and angiography. On dynamic CT scans, pancreatic AVM may exhibit strong enhancement and/or the conglomeration of small vascular spots with early contrast filling of the portal vein during the arterial phase (13). Angiography is most reliable tool for making the definitive diagnosis of pancreatic AVM and is useful for providing subsequent transcatheter therapy. The angiographic findings of pancreatic AVM are characterized by the detection of dilated and tortuous feeding arteries, a racemose intratumoral vascular network followed by transient dense pancreatic staining, early filling of the draining veins, such as the portal vein, during the arterial phase and the early disappearance of pancreatic staining $(13,14)$.

With respect to the management of pancreatic AVM, surgical therapy and conservative modalities, such as TAE, irradiation, transjugular intrahepatic portosystemic shunt (TIPS) or treatment of esophageal varices, have been applied $(1,3,8)$. Although surgical resection is the most common treatment modality and usually achieves a complete cure, it is not always feasible due to difficulties during surgery based on limitations regarding the size, location and accessibility of the AVM and/or the risk of massive hemorrhage (15). Parsaik et al. reported a perioperative mortality 

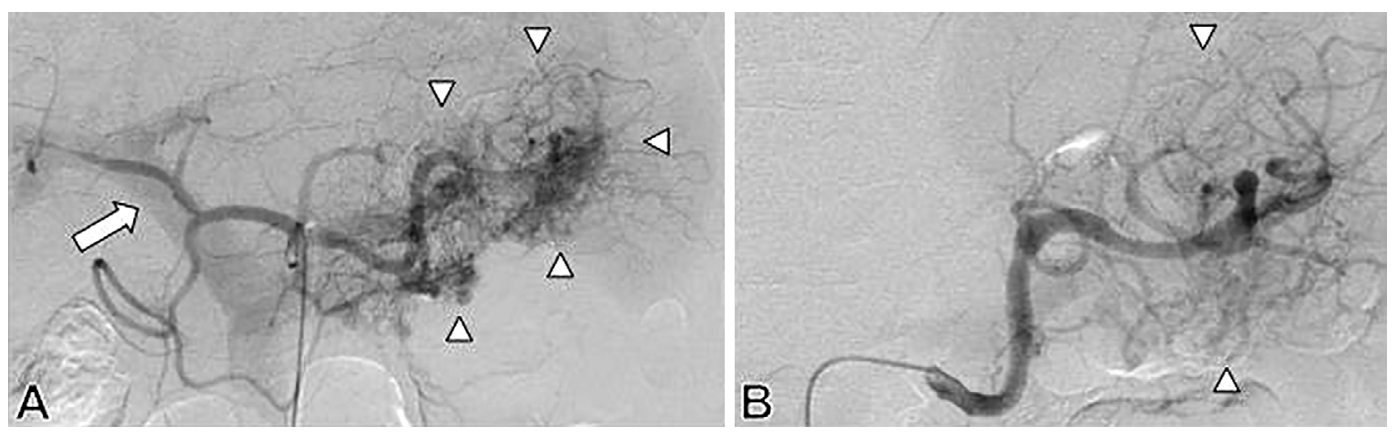

Figure 2. (A) Angiography of the celiac artery showed racemose vascular networks in the body and tail of the pancreas (arrowheads), with an early venous return to the portal vein (arrow). (B) A fraction of the pancreatic AVM remained after embolization (arrowheads); however, no early venous return to the portal vein was detected.
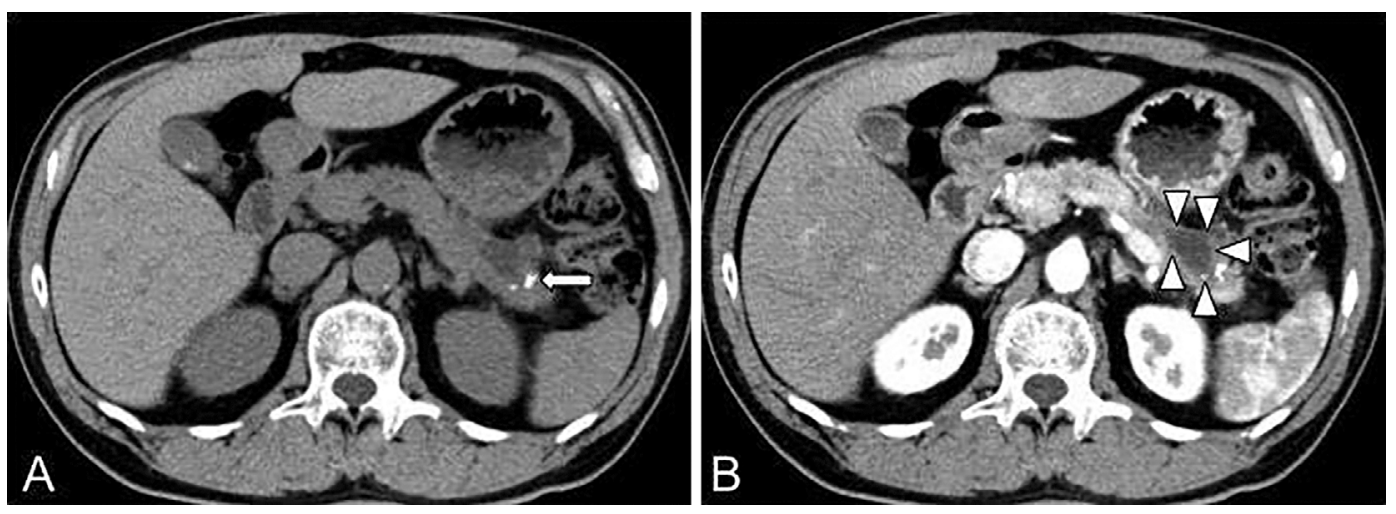

Figure 3. CT scan obtained six months after embolization. (A) Non-contrast-enhanced CT showed retention of the embolic agent (arrow). (B) Contrast-enhanced CT showed an embolized pancreatic AVM presenting as a low-density lesion (arrowheads), with no residual AVM in the pancreas.

Table. Summary of 13 Pancreatic AVM Cases Treated with TAE

\begin{tabular}{llll}
\hline & $\begin{array}{l}\text { TAE alone } \\
(\mathrm{n}=7)\end{array}$ & $\begin{array}{l}\text { TAE and surgery } \\
(\mathrm{n}=6)\end{array}$ & p value \\
\hline $\begin{array}{l}\text { Hemorrhage } \\
\text { Absence }\end{array}$ & $5(71.4 \%)$ & 0 & 0.008 \\
Presence & $2(28.6 \%)$ & $6(100 \%)$ & \\
\hline
\end{tabular}

Categorical value was compared using Fisher's exact probability test. p value less than 0.05 was considered statistically significant. TAE: transcatheter arterial embolization

rate of $5 \%$ for total pancreatectomy and postoperative problems (16).

TAE is a valuable alternative treatment for high-risk patients who are not candidates for surgical resection and as an initial therapy for life-threating gastrointestinal bleeding, the most common complication of pancreatic AVM (17). Furthermore, preoperative TAE is useful and effective for reducing the incidence of intraoperative bleeding, thereby improving surgical outcomes (18). However, the outcome of TAE can be unpredictable, as it is very difficult to embolize all of the multiple feeding arteries draining into the pancreatic AVM. Recurrent bleeding after successful TAE has been reported in up to $18-37 \%$ of patients waiting for sur- gery $(19,20)$. Thirteen previous case reports of pancreatic AVM treated with TAE are summarized in Table (3-5, 17, 19, 21-28). Cases successfully treated with TAE alone were more common among patients without hemorrhage than those with hemorrhage. In contrast, all patients with hemorrhage treated with TAE required surgery. From this point of view, we consider that our patient was cured with TAE alone without surgery because he had no history of bleeding due to the pancreatic AVM. In this case, TAE with NBCA was effective in relieving the patient's abdominal pain by resolving the pancreatic AVM. As to embolic agents for TAE, metallic coils, gelatin sponges and liquid agents are commonly used in patients with AVMs in various organs. However, due to the small number of patients, the effects of each embolic agent applied in TAE for pancreatic AVM are not well investigated. Liquid embolic agents such as NBCA and ethylene vinyl alcohol (EVOH) copolymer can be used to embolize vessels in the more distal vasculature by traveling within the feeding arteries of the AVM, in comparison to that achieved with metallic coils or gelatin sponges $(4,29)$. NBCA and EVOH are commonly used in TAE for AVMs in various organs, including the brain, kidney, uterus and extremities (30-35). In cases of renal AVMs, TAE with NBCA is more effective than TAE with coils alone (32). Regarding 
the pancreas, TAE with NBCA has been safely applied in an animal model (36). Therefore, in recently reported cases of pancreatic AVM, NBCA or EVOH has been used for TAE in combination with or without other embolic agents $(3,4)$. However, it is important to be aware of the risk of complications caused by embolization with NBCA, such as bowel ischemia resulting from distal organ embolization $(37,38)$. Therefore, in the present case, a balloon catheter was used for embolization in order to prevent reflux of the NBCA to the distal vascular bed. The current patient has been followed for only six months and requires long-term follow-up. If the pancreatic AVM recurs, appropriate therapies will be considered based on the clinical features of AVM and the patient's general condition.

In summary, the current patient had a pancreatic AVM that was successfully treated using TAE with NBCA without major complications related to distal organ embolization. Although the characteristics of the AVM, especially the presence or absence of hemorrhage, generally account for the outcomes of TAE in patients with pancreatic AVMs, embolic agents may also be important factors influencing the effects of TAE.

\section{The authors state that they have no Conflict of Interest (COI).}

\section{References}

1. Chou SC, Shyr YM, Wang SE. Pancreatic arteriovenous malformation. J Gastrointest Surg 17: 1240-1246, 2013.

2. Kanno A, Satoh K, Kimura K, et al. Acute pancreatitis due to pancreatic arteriovenous malformation: 2 case reports and review of the literature. Pancreas 32: 422-425, 2006.

3. Kodama Y, Saito H, Hiramatsu K, Takeuchi S, Takamura A. A case of pancreatic arteriovenous malformation treated by transcatheter arterial embolization and transjugular intrahepatic portosystemic shunt. Nihon Shokakibyo Gakkai Zasshi (Jpn J Gastroenterol) 98: 320-324, 2001 (in Japanese).

4. Grasso RF, Cazzato RL, Luppi G, et al. Pancreatic arteriovenous malformation involving the duodenum embolized with ethylenevinyl alcohol copolymer (Onyx). Cardiovasc Intervent Radiol 35: 958-962, 2012.

5. Gomes AS, Busuttil RW, Baker JD, Oppenheim W, Machleder HI, Moore WS. Congenital arteriovenous malformations. The role of transcatheter arterial embolization. Arch Surg 118: 817-825, 1983.

6. Halpern M, Turner AF, Citron BP. Hereditary hemorrhagic telangiectasia. An angiographic study of abdominal visceral angiodysplasias associated with gastrointestinal hemorrhage. Radiology 90: 1143-1149, 1968.

7. Arora A, Tyagi P, Kirnake V, et al. Unusual cause of massive upper gastrointestinal bleeding: a pancreatic arteriovenous malformation. JOP 10: 292-295, 2013.

8. Shimizu K, Sunagawa Y, Ouchi K, Mogami T, Harada J, Fukuda $\mathrm{K}$. External beam radiotherapy for angiographically diagnosed arteriovenous malformation involving the entire pancreas. Jpn J Radiol 31: 760-765, 2013.

9. Suenaga M, Fujii T, Kanda M, et al. Recurrent acute pancreatitis due to arteriovenous malformation of the pancreas. Nihon Shokakibyo Gakkai Zasshi (Jpn J Gastroenterol) 110: 1983-1988, 2013 (in Japanese, Abstract in English).

10. Yamabuki T, Ohara $M$, Kimura $N$, et al. Pancreatic arteriovenous malformation. Case Rep Gastroenterol 8: 26-31, 2014.
11. Nishiyama R, Kawanishi $Y$, Mitsuhashi $H$, et al. Management of pancreatic arteriovenous malformation. J Hepatobiliary Pancreat Surg 7: 438-442, 2000.

12. Butte JM, San Francisco IF, Pacheco F, Solar A, Crovari FJ, Jarufe NP. Arteriovenous malformation of the pancreas: report of a case. Surg Today 37: 604-607, 2007.

13. Chang S, Lim HK, Lee WJ, Choi D, Jang KT. Arteriovenous malformation of the pancreas in a patient with gastrointestinal bleeding: helical CT findings. Abdom Imaging 29: 259-262, 2004.

14. Chuang VP, Pulmano CM, Walter JF, Cho KJ. Angiography of pancreatic arteriovenuos malformation. Am J Roentgenol 129: 1015-1018, 1977.

15. Song KB, Kim SC, Park JB, et al. Surgical outcomes of pancreatic arteriovenous malformation in a single center and review of literature. Pancreas 41: 388-396, 2012.

16. Parsaik AK, Murad MH, Sathananthan A, et al. Metabolic and target organ outcomes after total pancreatectomy: Mayo Clinic experience and meta-analysis of the literature. Clin Endocrinol (Oxf) 73: 723-731, 2010.

17. Makhoul F, Kaur P, Johnston TD, Jeon H, Gedaly R, Ranjan D. Arteriovenous malformation of the pancreas: a case report and review of literature. Int J Angiol 17: 211-213, 2008.

18. Rezende MB, Bramhall S, Hayes T, et al. Pancreatic arteriovenous malformation. Dig Surg 20: 65-69, 2003.

19. Uda O, Aoki T, Tsuchida A, et al. Pancreatic arteriovenous malformation observed to bleed from the bile duct and a duodenal ulcer: report of a case. Surg Today 29: 462-466, 1999.

20. Zyromski NJ, Vieira C, Stecker M, et al. Improved outcomes in postoperative and pancreatitis-related visceral pseudoaneurysms. J Gastrointest Surg 11: 50-55, 2007.

21. Charalabopoulos A, Macheras N, Krivan S, Petropoulos K, Misiakos E, Macheras A. Arteriovenous malformation of the pancreas. Case Rep Med 2011: 612657, 2011.

22. Sharma M, Bedi MM, Mahesh S, et al. Arteriovenous malformation of the pancreatic head: difficulties in diagnosis and treatment. Indian J Gastroenterol 30: 46-48, 2011.

23. Gincul R, Dumortier J, Ciocirlan M, et al. Treatment of arteriovenous malformation of the pancreas: a case report. Eur J Gastroenterol Hepatol 22: 116-120, 2011.

24. Perera MT, Shimoda M, Kato M, et al. Life-threatening bleeding from duodenal varices due to pancreatic arterio-venous malformation: role of emergency pancreatoduodenectomy. Hepatogastroenterology 55: 1553-1556, 2008.

25. Yamamoto K, Tominaga K, Kanke K, et al. Education and imaging. Hepatobiliary and pancreatic: arteriovenous malformation of the pancreas. J Gastroenterol Hepatol 23: 498, 2008.

26. Hosogi H, Ikai I, Hatano E, et al. Pancreatic arteriovenous malformation with portal hypertension. J Hepatobiliary Pancreat Surg 13: 344-346, 2006.

27. Iwashita Y, Kawano T, Maeda T, Nagasaki S, Kitano S. Pancreatic arteriovenous malformation treated by transcatheter embolization. Hepatogastroenterology 49: 1722-1723, 2002.

28. Kato T, Takahashi M, Okawada T, Miyazaki Y, Kaneko M. Pancreatic arteriovenous malformation treated by transcatheter embolization: report of a case with hepatocellular carcinoma. Radiat Med 9: 19-21, 1991.

29. Leyon JJ, Littlehales T, Rangarajan B, Hoey ET, Ganeshan A. Endovascular embolization: review of currently available embolization agents. Curr Probl Diagn Radiol 43: 35-53, 2014.

30. $\mathrm{Lv} \mathrm{X}, \mathrm{Wu} \mathrm{Z}, \mathrm{Li}$ Y. Arteriovenous malformation in the brain: a theoretical study explaining the behavior of liquid embolic agents during endovascular treatment. Neuroradiol J 26: 661-668, 2013.

31. Pierot L, Cognard C, Herbreteau D, et al. Endovascular treatment of brain arteriovenous malformations using a liquid embolic agent: results of a prospective, multicentre study (BRAVO). Eur Radiol 23: 2838-2845, 2013. 
32. Zhang B, Jiang ZB, Huang MS, Zhu KS, Guan SH, Shan H. The role of transarterial embolization in the management of hematuria secondary to congenital renal arteriovenous malformations. Urol Int 91: 285-290, 2013.

33. Murata S, Onozawa S, Nakazawa K, et al. Endovascular embolization strategy for renal arteriovenous malformations. Acta Radiol 55: 71-77, 2014.

34. Molvi SN, Dash K, Rastogi H, Khanna SB. Transcatheter embolization of uterine arteriovenous malformation: report of 2 cases and review of literature. J Minim Invasive Gynecol 18: 812-819, 2011.

35. Kitagawa A, Izumi $Y$, Hagihara $M$, et al. Ethanolamine oleate sclerotherapy combined with transarterial embolization using $n$ butyl cyanoacrylate for extracranial arteriovenous malformations. Cardiovasc Intervent Radiol 37: 371-380, 2014.
36. Okada $\mathrm{T}$, Yamaguchi $\mathrm{M}$, Takahashi $\mathrm{T}$, et al. Is embolization of the pancreas safe? Pancreatic histological changes after selective transcatheter arterial embolization with $\mathrm{N}$-butyl cyanoacrylate in a swine model. Cardiovasc Intervent Radiol 35: 161-167, 2012.

37. Jae HJ, Chung JW, Kim HC, et al. Experimental study on acute ischemic small bowel changes induced by superselective embolization of superior mesenteric artery branches with N-butyl cyanoacrylate. J Vasc Interv Radiol 19: 755-763, 2008.

38. Ogawa Y, Takizawa K, Funakubo M, Yagihashi K, Nakajima Y, Tadokoro M. A Histological study in an animal model after embolization of the mesenteric artery with N-butyl-2-cyanoacrylate. Nihon Fukubu Kyukyu Igakkai Zasshi (J Abdomin Emerg Med) 28: 775-780, 2008 (in Japanese, Abstract in English).

(C) 2014 The Japanese Society of Internal Medicine http://www.naika.or.jp/imonline/index.html 